\title{
Iterated Darboux Transformation for Isothermic Surfaces in Terms of Clifford Numbers
}

\author{
Jan L. Cieśliński *(D) and Zbigniew Hasiewicz (D) \\ Wydział Fizyki, Uniwersytet w Białymstoku, ul. Ciołkowskiego 1L, 15-245 Białystok, Poland; \\ z.hasiewicz@uwb.edu.pl \\ * Correspondence: j.cieslinski@uwb.edu.pl
}

Citation: Cieśliński, J.L.; Hasiewicz,

Z. Iterated Darboux Transformation for Isothermic Surfaces in Terms of Clifford Numbers. Symmetry 2021, 13, 148. https://doi.org/10.3390/sym 13010148

Received: 31 December 2020

Accepted: 15 January 2021

Published: 17 January 2021

Publisher's Note: MDPI stays neutral with regard to jurisdictional clai$\mathrm{ms}$ in published maps and institutional affiliations.

Copyright: () 2021 by the authors. Licensee MDPI, Basel, Switzerland. This article is an open access article distributed under the terms and conditions of the Creative Commons Attribution (CC BY) license (https:// creativecommons.org/licenses/by/ $4.0 /)$.

\begin{abstract}
Isothermic surfaces are defined as immersions with the curvture lines admitting conformal parameterization. We present and discuss the reconstruction of the iterated Darboux transformation using Clifford numbers instead of matrices. In particulalr, we derive a symmetric formula for the two-fold Darboux transformation, explicitly showing Bianchi's permutability theorem. In algebraic calculations an important role is played by the main anti-automorphism (reversion) of the Clifford algebra $\mathcal{C}(4,1)$ and the spinorial norm in the corresponding Spin group.
\end{abstract}

Keywords: integrable systems; Darboux-Bäcklund transformation; isothermic immersions; Spin groups; Clifford algebras

\section{Introduction}

Isothermic surfaces have a very long history. They have been first introduced by Lamé in studies on stationary heat flows (described by the Laplace equation), in the broader context of triply ortogonal systems of coordinates [1]. Then, the main progress towards the theory of isothermic surfaces was done by Bertrand [2], who was first to notice that "in any triply isothermic (in physical sense) orthogonal system in $\mathbb{E}^{3}$ any coordinate surface admits conformal curvature parameterization" [3]. Transformations of isothemic surfaces, studied by Darboux and Bianchi [4,5], strongly suggested that the related system of nonlinear partial differential equations (see (2) below) is integrable in the sense of the soliton theory [6] and, indeed, such modern formulation of this problem was found [7], which started new developments in this field [8-12]. It is worthwhile to mention that isothermic immersions are invariant with respect to conformal transformations of the ambient space and can be naturally described in terms of conformal geometry (then Darboux transformations correspond to Ribaucour congruences [13]). Studies on isothermic surfaces are still active, see, e.g., [14-19]. In this paper we develop an approach based on using Clifford algebras and Spin groups [20,21] (different from the approach of [12,22]). We re-derive the construction of "multisoliton" surfaces by iterated Darboux transformation. In particular, we present detailed computation of the two-fold Darboux transform.

\section{Isothermic Surfaces in $\mathbb{R}^{3}$}

Isothermic surfaces (or, more precisely, isothermic immersions) are characterized as surfaces immersed in $E^{3}$ with curvature lines admitting conformal parameterization. It means that there exist coordinates $(u, v)$ in which the isothermic immersion has the following fundamental forms:

$$
\begin{aligned}
& I=e^{2 \vartheta}\left(d u^{2}+d v^{2}\right), \\
& I I=e^{2 \vartheta}\left(k_{1} d u^{2}+k_{2} d v^{2}\right),
\end{aligned}
$$


where $\vartheta, k_{1}, k_{2}$ are functions of $u, v$, which have to satisfy the following system of nonlinear partial differential equations known as Gauss-Mainardi-Codazzi equations:

$$
\begin{aligned}
& \vartheta_{, u u}+\vartheta_{, v v}+k_{1} k_{2} e^{2 \vartheta}=0, \\
& k_{2, u}+\left(k_{2}-k_{1}\right) \vartheta_{, u}=0, \\
& k_{1, v}+\left(k_{1}-k_{2}\right) \vartheta_{, v}=0,
\end{aligned}
$$

where comma denotes partial derivtive. Geometrically, $k_{1}$ and $k_{2}$ are principal curvatures, and their product $k_{1} k_{2}$ yields the Gaussian curvature. The above nonlinear system can be obtained (see [7]) as compatibility conditions for the following linear problem (or Lax pair):

$$
\begin{aligned}
& \Psi_{, u}=\frac{1}{2} \mathbf{e}_{1}\left(-\vartheta, v \mathbf{e}_{2}+k_{1} e^{\vartheta} \mathbf{e}_{3}+\lambda \sinh \vartheta \mathbf{e}_{4}+\lambda \cosh \vartheta \mathbf{e}_{5}\right) \Psi, \\
& \Psi_{, v}=\frac{1}{2} \mathbf{e}_{2}\left(-\vartheta, u \mathbf{e}_{1}+k_{2} e^{\vartheta} \mathbf{e}_{3}+\lambda \cosh \vartheta \mathbf{e}_{4}+\lambda \sinh \vartheta \mathbf{e}_{5}\right) \Psi,
\end{aligned}
$$

where $\mathbf{e}_{1}, \ldots, \mathbf{e}_{5}$ are $4 \times 4$ complex matrices (for their exact form see [7] or [23]) that satisfy the relations

$$
\mathbf{e}_{1}^{2}=\mathbf{e}_{2}^{2}=\mathbf{e}_{3}^{2}=\mathbf{e}_{4}^{2}=-\mathbf{e}_{5}^{2}=1, \quad \mathbf{e}_{j} \mathbf{e}_{k}=-\mathbf{e}_{k} \mathbf{e}_{j} \quad(j \neq k) .
$$

We point out that using a suitable extension of the famous Sym formula (see, e.g., [24]) we can reconstruct the radius vector of the isothermic surface implicitly determined by the fundamental forms (1), for more details see Section 4 and Theorem 1.

\section{Clifford Algebras}

The matrices $\mathbf{e}_{1}, \ldots, \mathbf{e}_{5}$ satisfying (4) can be interpreted as elements of a Clifford algebra, see below. Their exact matrix form is not needed. From technical point of view, it is even easier to use Clifford numbers instead of particular matrix representations [21].

We recall the definition of a Clifford algebra generated by vectors of a Euclidean or pseudo-Euclidean inner product space. Let $V$ be a vector space endowed with a non-degenerate (but not necessarily positive definite) quadratic form $Q$, see, e.g., [25,26]. If $Q$ is positive definite (the Euclidean case), then $Q(v)$ is the square of the length of $v$. The associated bilinear form (scalar product) will be denoted by brackets. In particular, $Q(v) \equiv\langle v \mid v\rangle$. The Clifford algebra $\mathcal{C}(V, Q)$ is generated by products ("Clifford products") of vectors (elements of $V$ ). The Clifford product is defined by the following relation:

$$
v w+w v=2\langle v \mid w\rangle \mathbf{1}
$$

where 1 denotes the unit of the Clifford algebra.

Let $\left\{\mathbf{e}_{1}, \ldots, \mathbf{e}_{n}\right\}$ be an orthonormal basis of $V$, i.e., $\left\langle\mathbf{e}_{j} \mid \mathbf{e}_{k}\right\rangle=0$ for $j \neq k$, and $\left\langle\mathbf{e}_{k} \mid \mathbf{e}_{k}\right\rangle= \pm 1$. The dimension of the Clifford algebra is $2^{n}$. Its standard basis consists of

$$
\mathbf{1}, \quad \mathbf{e}_{k}, \quad \mathbf{e}_{j k}(j<k), \quad \mathbf{e}_{i} \mathbf{e}_{j} \mathbf{e}_{k}(i<j<k), \ldots
$$

If the signature of $Q$ is $(m, p)$ (i.e., among $\mathbf{e}_{1}, \ldots, \mathbf{e}_{n}$ there are $m$ vectors such that $\mathbf{e}_{j}^{2}=1$ and $p$ vectors such that $\mathbf{e}_{j}^{2}=-1$, and $\left.m+p=n\right)$, then we denote $\mathcal{C}(V, Q) \equiv \mathcal{C}_{m, p}$.

The Clifford group (or Lipschitz group) $\Gamma(V, Q)$ is the multiplicative group (with respect to the Clifford product) generated by the non-isotropic vectors (we recall that $w \in V$ is isotropic (or null) vector if $\langle w \mid w\rangle=0$ ). The spinor norm of an element $X \in \Gamma(V, Q)$ is defined as

$$
N(X):=\beta(X) X,
$$


where $\beta$ is a reversion, i.e., $\beta\left(v_{1} v_{2} \ldots v_{M}\right)=v_{M} v_{M-1} \ldots v_{2} v_{1}$. One can easily see that the spinor norm of a vector is its scalar square, $N(v)=\langle v \mid v\rangle$, and for any element of $\Gamma(V, Q)$ we have

$$
N\left(v_{1} v_{2} \ldots v_{M}\right)=\left\langle v_{1} \mid v_{1}\right\rangle\left\langle v_{2} \mid v_{2}\right\rangle \ldots\left\langle v_{M} \mid v_{M}\right\rangle .
$$

The group $\operatorname{Pin}(V, Q)$ is a subgroup of $\Gamma(V, Q)$ consisting of elements $X$ such that $N^{2}(X)= \pm 1$ (i.e., $\Gamma(V, Q)$ contains products of unit vectors), and the group $\operatorname{Spin}(V, Q)$ (a subgroup of $\operatorname{Pin}(V, Q)$ ) consists of products of even number of unit vectors [26].

\section{Spin-Valued Lax Pairs}

Our approach to the construction of Darboux transformation consists of two steps. First, we characterize the structure of the Lax pair. Second, we are looking for a transformation preserving the structure [27].

The structure of the Lax pair is characterized by the dependence on $\lambda$ (e.g., divisor of poles) [6,28], reduction group (loop group) [29] and, possibly, by other invariants of Darboux transformations, like linear and multilinear constraints on coefficients of the Laurent expansion around poles [30].

In this section we present the characterization of the Lax pair (3), see [20,31]. First of all, we consider Clifford numbers instead of matrices. Then, we notice that both matrices of this Lax pair are Clifford bi-vectors linear in $\lambda$ such that

$$
\Psi_{, \mu}=U_{\mu} \Psi, \quad U_{\mu}=\frac{1}{2} \mathbf{e}_{\mu}\left(\lambda \mathbf{a}_{\mu}+\mathbf{b}_{\mu}\right) \quad(\mu=1,2)
$$

where $\Psi=\Psi(u, v), \Psi_{, 1} \equiv \Psi_{, u}, \Psi_{, 2} \equiv \Psi_{, v}, \mathbf{a}_{\mu}=\mathbf{a}_{\mu}(u, v) \in W, \mathbf{b}_{\mu}=\mathbf{b}_{\mu}(u, v) \in V, V$ and $W$ are real vector spaces, $V$ is spanned by $\mathbf{e}_{1}, \mathbf{e}_{2}$ and $\mathbf{e}_{3}$, and $W$ is spanned by $\mathbf{e}_{4}$ and $\mathbf{e}_{5}$. We assume relations (4), which means that form now on the quadratic form $Q$, defined on $V \oplus W$, is assumed to have signature $(4,1)$.

The compatibility conditions for the linear system (8) imply that $\mathbf{a}_{1}$ and $\mathbf{a}_{2}$ form an orthogonal basis in $W$. We can confine ourselves to the particular case (3) without loss of the generality. Indeed, both linear problems are equivalent up to a re-parameterization of independent variables and a discrete transformation in the space $W$.

The form (8) of the spectral problem can be described in terms of some group constraints ("reduction group", compare $[27,29]$ ). First, $U_{\mu}$ are linear combinations of Clifford bi-vectors. In other words, $U_{\mu}$ take values in the Lie algebra of the group $\operatorname{Spin}(V \oplus W, Q)$. In principle, $\Psi$ could be a spinor, but here and in the sequel we assume that it is an element of the Clifford algebra. Without loss of the generality we can confine ourselves to solutions $\Psi \in \Gamma(V, Q)$. The next observation is $\beta\left(U_{\mu}\right)=-U_{\mu}$.

Lemma 1. If $\beta\left(U_{\mu}\right)=-U_{\mu}($ for $\mu=1,2)$, and $\Psi$ satisfies $\Psi_{, \mu}=U_{\mu} \Psi$, then

$$
N(\Psi) \equiv \Psi \beta(\Psi)=\text { const }
$$

Proof. It is sufficient to differentiate $N(\Psi)$ :

$$
(N(\Psi))_{, \mu}=\Psi, \mu \boldsymbol{\beta}(\Psi)+\Psi \boldsymbol{\beta}(\Psi, \mu)=\left(U_{\mu}+\boldsymbol{\beta}\left(U_{\mu}\right)\right) N(\Psi)=0,
$$

where one has to remember that $N(\Psi)$ is a scalar, so it commutes with any elements.

Therefore, $\Psi \in \operatorname{Spin}(V \oplus W, Q)$ (for any $u, v$ ) provided that $\Psi$ is Spin-valued at some initial point $\left(u_{0}, v_{0}\right)$. In an analogous way one can show the following loop group conditions:

$$
\Psi(-\lambda)=\mathbf{e}_{4} \mathbf{e}_{5} \Psi(\lambda) \mathbf{e}_{4} \mathbf{e}_{5}, \quad \overline{\Psi(\lambda)}=\Psi(\bar{\lambda})
$$

(where the bar denotes complex conjugate and, by definition, $\overline{\mathbf{e}}_{j}=\mathbf{e}_{j}$ ). They follow from

$$
\mathbf{e}_{4} \mathbf{e}_{5} U_{\mu}(\lambda)=U_{\mu}(-\lambda) \mathbf{e}_{4} \mathbf{e}_{5}, \quad \overline{U_{\mu}(\lambda)}=U_{\mu}(\bar{\lambda})
$$


The properties $\beta\left(U_{\mu}\right)=-U_{\mu}$ and (12) hold for the Lax pair (8), which can be easily verified using commutation relations (4).

The Sym-Tafel formula $F=2 \Psi^{-1} \Psi_{\lambda}$ [24], evaluated in $\lambda=0$, yields a surface immersed into the 6-dimensional space spanned by bi-vectors of the form $\mathbf{e}_{k} \mathbf{e}_{\alpha}(k=1,2,3$; $\alpha=4,5$ ). Projecting this surface on especially chosen 3 -dimensional subspaces we obtain the original isothermic surface as a linear combination of $\mathbf{e}_{k}\left(\mathbf{e}_{4}+\mathbf{e}_{5}\right)(k=1,2,3)$ and its dual (or Christoffel transform) as a combination of $\mathbf{e}_{k}\left(\mathbf{e}_{4}-\mathbf{e}_{5}\right)(k=1,2,3)$ [23].

Here we present some details of calculations involving Clifford numbers instead of matrices. They are closely related but not identical to the approach of our earlier papers $[20,23,32]$.

We use the projection $P: \mathcal{C}(V \oplus W) \rightarrow \mathcal{C}(V)$ defined as a homomorphism of Clifford algebras such that

$$
P\left(\mathbf{e}_{4}\right)=P\left(\mathbf{e}_{5}\right)=1 .
$$

Note that

$$
P\left(\sum_{j=1}^{3} \sum_{\alpha=4}^{5} c_{j \alpha} \mathbf{e}_{j} \mathbf{e}_{\alpha}\right)=\sum_{j=1}^{3}\left(c_{j 4}+c_{j 5}\right) \mathbf{e}_{j}
$$

This projection yields an original isothermic surface (the dual surface is a result of another homomorphism $P^{\prime}$, defined by $\left.P^{\prime}\left(\mathbf{e}_{4}\right)=-P^{\prime}\left(\mathbf{e}_{5}\right)=1\right)$.

Theorem 1. We assume that $\Psi$ satisfies the linear system (3) and

$$
F:=2 \Psi^{-1} \Psi,\left.\lambda\right|_{\lambda=0} .
$$

Then $\mathbf{r}:=P(F)$ yields the original isothermic immersion (up to a Euclidean motion), provided that we identify span $\left\{\mathbf{e}_{1}, \mathbf{e}_{2}, \mathbf{e}_{3}\right\}$ with the space $\mathbb{E}^{3}$.

Proof. The crucial property of the Sym-Tafel formula (15) is a compact form of its derivative (compare [24]):

$$
F_{\mu}=2 \Psi_{0}^{-1} U_{\mu, \lambda}(0) \Psi_{0}=\Psi_{0}^{-1} \mathbf{e}_{\mu} \mathbf{a}_{\mu} \Psi_{0},
$$

where $\Psi_{0}:=\Psi(0)$ (i.e., $\Psi$ evaluated at $\lambda=0$ ), and we use the slightly more general form (8) of the Lax pair (3). Then

$$
\mathbf{r}_{, \mu}=e^{\theta} \Psi_{0}^{-1} \mathbf{e}_{\mu} \Psi_{0} .
$$

Therefore

$$
E_{\mu}=\Psi_{0}^{-1} \mathbf{e}_{\mu} \Psi_{0}
$$

(for $\mu=1,2$ ) form an orthonormal basis in the tangent space and, therefore, the corresponding metric is given by the first equation of (1). Obviously, $E_{3}$ (defined by (18) for $\mu=3$ ) is orthogonal to $E_{1}$ and $E_{2}$; hence, it can be identified with the normal vector. In order to derive the second fundamental form we differentiate (17). Taking into account $\Psi_{0, \mu}=\frac{1}{2} e_{\mu} b_{\mu} \Psi_{0}$, we obtain

$$
\begin{aligned}
& \mathbf{r}_{11}=e^{\theta}\left(\theta, 1 E_{1}+\left\langle b_{1} \mid \mathbf{e}_{2}\right\rangle E_{2}+\left\langle b_{1} \mid \mathbf{e}_{3}\right\rangle E_{3}\right)=e^{\theta}\left(\theta, 1 E_{1}-\theta_{, 2} E_{2}+k_{1} e^{\theta} E_{3}\right), \\
& \mathbf{r}_{12}=e^{\theta}\left(\theta, 2 E_{1}-\left\langle b_{2} \mid \mathbf{e}_{1}\right\rangle E_{2}\right)=e^{\theta}\left(\theta_{, 2} E_{1}+\theta, E_{2}\right), \\
& \mathbf{r}_{21}=e^{\theta}\left(-\left\langle b_{1} \mid \mathbf{e}_{2}\right\rangle E_{1}+\theta, 1 E_{2}\right)=e^{\theta}\left(\theta_{, 2} E_{1}+\theta_{, 1} E_{2}\right), \\
& \mathbf{r}_{, 22}=e^{\theta}\left(\left\langle b_{2} \mid \mathbf{e}_{1}\right\rangle E_{1}+\theta_{, 2} E_{2}+\left\langle b_{2} \mid \mathbf{e}_{3}\right\rangle E_{3}\right)=e^{\theta}\left(-\theta_{, 1} E_{1}+\theta_{, 2} E_{2}+k_{2} e^{\theta} E_{3}\right) .
\end{aligned}
$$

Therefore, coefficients of the second fundamental form (given by $\left\langle\mathbf{r}_{i j} \mid E_{3}\right\rangle$ ) yield the second formula of (1). The proof is completed by applying the Bonnet theorem. 


\section{The Darboux-Bäcklund Transformation in the Case of Spin Groups}

The Darboux transformation is a gauge-like transformation using the Darboux matrix $D$ (we will keep using the name "matrix" even for $D$ given in terms of Clifford numbers without referring to any matrix representation):

$$
\tilde{\Psi}=D \Psi, \quad \tilde{\Psi}_{, \mu}=\tilde{U}_{\mu} \tilde{\Psi}, \quad \tilde{U}_{\mu}=D_{, \mu} D^{-1}+D U_{\mu} D^{-1},
$$

provided that $\tilde{U}_{\mu}$ has the same dependence on dependent variables as $U_{\mu}$, see, e.g., [6,33]. In Section 4 we have shown that the form (3) can be derived by imposing a set of constraints on a general linear problem $\left(U_{\mu}\right.$ are Clifford bi-vectors, linear in $\lambda$ and belong to the appropriate loop algebra). Then the Darboux transformation has to preserve this structure, which means, in particular, that $D$ should belong to the same group as $\Psi$.

Different methods of constructing the Darboux matrix need different form of $\lambda$-dependence of $D$ (these forms are equivalent up to a $\lambda$-dependent scalar factor [30]). In particular, one can assume $D$ as polynomial in $\lambda$ (eigenvalues, corresponding to solitons, are zeros of $\operatorname{det} D$ ) [34], sum of simple fractions (eigenvalues: poles of $D$ and $D^{-1}$ ) [6,29], or a "realization" $\left(D=N+F(\lambda-A)^{-1} G\right)[35,36]$.

Our motivation for dealing with the case of Spin groups came from yet another approach [31]. Multiplying (20) by $D^{2}(\lambda)$ we get

$$
D, \mu D+D U_{\mu} D=\tilde{U}_{\mu} D^{2} .
$$

It is a crucial point that the right-hand side vanishes for $\lambda_{+}$and $\lambda_{-}$such that $D^{2}\left(\lambda_{ \pm}\right)=0$. Then, we obtain a solution of the remaining equation: $D\left(\lambda_{ \pm}\right)=\rho_{ \pm} \Psi\left(\lambda_{ \pm}\right) d_{ \pm}$ $\Psi\left(\lambda_{ \pm}\right)^{-1}$, where $d_{ \pm}=$const, $\left(d_{ \pm}\right)^{2}=0$ and $\rho_{ \pm}$are two scalar functions. Finally, $D(\lambda)$ is given as a linear combination of $D\left(\lambda_{+}\right)$and $D\left(\lambda_{-}\right)$with coefficients linear in $\lambda$ [31], which yields one-soliton Darboux matrix. This approach was extended on the multi-soliton case for $2 \times 2$ matrix problems [37].

Generalization of this approach on Spin-valued linear problems is quite natural. Instead of multiplying both sides of (20) by $D^{2}$ we multiply them by $D \boldsymbol{\beta}(D)$ :

$$
D_{, \mu} \boldsymbol{\beta}(D)+D U_{\mu} \boldsymbol{\beta}(D)=\tilde{U}_{\mu} D \boldsymbol{\beta}(D) .
$$

Note that $\boldsymbol{\beta}(D)=D$ if $D$ is a Clifford vector (which has been usually assumed in earlier papers, like [31]), and in this case Equation (22) assumes the form (21).

Lemma 2. If an isotropic Clifford vector $D^{\prime}$ satisfies Equation (22), and $G$ is any Clifford number $G$ (not necessarily constant), then $D=G D^{\prime}$ satisfies Equation (22) as well.

Proof. Lemma can be shown by straightforward calculation. First, we have

$$
D \boldsymbol{\beta}(D)=G D^{\prime} \boldsymbol{\beta}\left(D^{\prime}\right) \boldsymbol{\beta}(G)=G\left(D^{\prime}\right)^{2} \boldsymbol{\beta}(G)=0,
$$

so the right-hand side of (22) vanishes. Then

$$
D_{, \mu} \boldsymbol{\beta}(D)+D U_{\mu} \boldsymbol{\beta}(D)=G_{, \mu} D^{\prime} \boldsymbol{\beta}\left(D^{\prime}\right) \boldsymbol{\beta}(G)+G\left(D^{\prime}{ }_{\mu} \boldsymbol{\beta}\left(D^{\prime}\right)+D^{\prime} U_{\mu} \boldsymbol{\beta}\left(D^{\prime}\right)\right) \boldsymbol{\beta}(G)=0,
$$

which ends the proof.

In this paper we confine ourselves to iterations of the simplest Darboux transformations (defined by $D$ linear in $\lambda$ ). Then we can use the results of [31], where the case of the Clifford vector (here denoted by $D^{\prime}$ ) was considered, and the following form of the Darboux transformation was derived:

$$
D^{\prime}(\lambda)=\frac{\lambda-\lambda_{-}}{\lambda_{+}-\lambda_{-}} D^{\prime}\left(\lambda_{+}\right)+\frac{\lambda-\lambda_{+}}{\lambda_{-}-\lambda_{+}} D^{\prime}\left(\lambda_{-}\right),
$$


and $D^{\prime}\left(\lambda_{ \pm}\right)$can be expressed as

$$
D^{\prime}\left(\lambda_{ \pm}\right)=\rho_{ \pm} \Psi\left(\lambda_{ \pm}\right) d_{ \pm} \Psi\left(\lambda_{ \pm}\right)^{-1}
$$

where $\rho_{ \pm}$are (arbitrary) scalar functions and $d_{ \pm}$are constant elements such that $d_{ \pm}^{2}=0$. Reductions (12) impose constraints on $\lambda_{ \pm}, \rho_{ \pm}$, and $d_{ \pm}$(see [31]):

$$
\lambda_{+}=i \kappa, \quad \lambda_{-}=-i \kappa \quad(\kappa \in \mathbb{R}), \quad \rho_{+}=\rho_{-} \equiv \rho \in \mathbb{R}, \quad d_{ \pm}=\kappa\left(p_{0} \pm i n_{0}\right) .
$$

Moreover we denote (compare [21])

$$
p+i n:=\Psi(i \kappa)\left(p_{0}+i n_{0}\right) \Psi^{-1}(i \kappa)
$$

We assume

$$
p_{0}^{2}=n_{0}^{2}=1
$$

(in the Clifford algebra $p^{2}=\langle p \mid p\rangle$ etc.). Therefore computing the Clifford square of both sides of (28) and taking into account that $p, p_{0}$ anticommute with $n, n_{0}$ we get $p^{2}=n^{2}$.

It is convenient to introduce unit vectors $\hat{p}$ and $\hat{n}$

$$
\hat{p}:=\frac{p}{\sqrt{\langle p \mid p\rangle}}, \quad \hat{n}:=\frac{n}{\sqrt{\langle n \mid n\rangle}},
$$

such that $\hat{p}^{2}=\hat{n}^{2}=1$. Then the Darboux matrix assumes the form $D^{\prime}(\lambda)=\lambda \hat{n}+\kappa \hat{p}$. In order to get a Spin-valued $D$ we can take, for instance, $G=\mathbf{e}_{4}$, obtaining

$$
D(\lambda):=\mathbf{e}_{4}(\lambda \hat{n}+\kappa \hat{p})
$$

Note that

$$
D(\lambda) \boldsymbol{\beta}(D(\lambda))=\lambda^{2}+\kappa^{2}
$$

Remark 1. It is important to remember that the obtained Darboux matrix $D$ depends on the function $\Psi$ (an exact solution of the linear problem (3)) and constant parameters: $\kappa, p_{0}, n_{0}$. The notation $D=D_{\left[\Psi, \kappa, p_{0}, n_{0}\right]}$ would be very awkward, so in the sequel we omit the dependence on $p_{0}$ and $n_{0}$, writing $D=D_{[\Psi, \kappa]}$.

Theorem 2. The transformation $\tilde{\Psi}(\lambda)=\mathbf{e}_{4}(\lambda \hat{n}+\kappa \hat{p}) \Psi(\lambda)$, where $\hat{n}$ and $\hat{p}$ are given by (29) and

$$
\begin{aligned}
& p:=\frac{1}{2}\left(\Psi(i \kappa)\left(p_{0}+i n_{0}\right) \Psi^{-1}(i \kappa)+\Psi(-i \kappa)\left(p_{0}-i n_{0}\right) \Psi^{-1}(-i \kappa)\right) \\
& n:=\frac{1}{2 i}\left(\Psi(i \kappa)\left(p_{0}+i n_{0}\right) \Psi^{-1}(i \kappa)-\Psi(-i \kappa)\left(p_{0}-i n_{0}\right) \Psi^{-1}(-i \kappa)\right),
\end{aligned}
$$

transforms the linear problem (3) into the linear problem of the same form with $\theta, k_{1}$ and $k_{2}$ replaced by

$$
\begin{aligned}
& \tilde{\theta}=\theta-2 \gamma, \\
& \tilde{k}_{1}=e^{2 \gamma}\left(k_{1}-2 \kappa\left\langle p \mid \mathbf{e}_{3}\right\rangle e^{\theta} \sinh (\theta-\gamma)\right), \\
& \tilde{k}_{2}=e^{2 \gamma}\left(k_{2}-2 \kappa\left\langle p \mid \mathbf{e}_{3}\right\rangle e^{\theta} \cosh (\theta-\gamma)\right),
\end{aligned}
$$

where $\gamma$ is a function parameterizing $\hat{n}$, namely: $\hat{n}=\cosh \gamma \mathbf{e}_{4}+\sinh \gamma \mathbf{e}_{5}$.

We omit the proof, which consists in splitting the equation $D_{, \mu}+D U_{\mu}=\tilde{U}_{\mu} D$ into a system of equations by equating coefficients by powers of $\lambda$ and basis elements of the Clifford algebra. 
Theorem 3. The Darboux transformation for soliton submanifolds (15) reads

$$
\tilde{F}=F+\frac{2}{\kappa} \hat{p}^{-1} \hat{n}, \quad \tilde{\mathbf{r}}=\mathbf{r}+\frac{2 e^{\gamma}}{\kappa} \hat{p} .
$$

Proof. Directly applying the Sym formula we get

$$
\tilde{F}=2(D \Psi)^{-1}(D \Psi),\left.\lambda\right|_{\lambda=0}=\left.2 \Psi^{-1} \Psi_{, \lambda}\right|_{\lambda=0}+\left.2 \Psi_{0}^{-1}\left(D^{-1} D_{, \lambda}\right)\right|_{\lambda=0} \Psi_{0} \text {. }
$$

Substituting $D_{, \lambda}=\hat{n}$ and $D^{-1}(0)=\kappa^{-1} \hat{p}^{-1}$, we get the first formula of (34). To obtain the second formula we take into account that $\hat{p}^{2}=1$ and $P(\hat{n})=e^{\gamma}$.

In the context of soliton surfaces the Darboux transformation is often called the Darboux-Bäcklund transformation [21,31] or the Darboux-Bianchi transformation [23].

\section{Iterated Darboux Transformation}

The Darboux transformation can be iterated in a natural way. Using the notation introduced in Remark 1 we have the following sequence of solutions to the considered linear problem:

$$
\begin{aligned}
& \Psi^{[1]}(\lambda)=D_{\left[\Psi[0], \kappa_{1}\right]}(\lambda) \Psi^{[0]}(\lambda), \\
& \Psi^{[2]}(\lambda)=D_{\left[\Psi[1], \kappa_{2}\right]}(\lambda) \Psi^{[1]}(\lambda) \\
& \ldots \ldots \ldots \ldots \ldots \ldots, \\
& \Psi[K](\lambda)=D_{\left[\Psi[K-1], \kappa_{K}\right]}(\lambda) \Psi^{[K-1]}(\lambda) .
\end{aligned}
$$

The last equation can be rewritten in the following, more explicit, way:

$$
\Psi^{[K]}(\lambda)=D_{\left[\Psi[K-1], \kappa_{K}\right]}(\lambda) D_{\left[\Psi[K-2], \kappa_{K-1}\right]}(\lambda) \ldots D_{\left[\Psi[1], \kappa_{2}\right]}(\lambda) D_{\left[\Psi[0], \kappa_{1}\right]}(\lambda) \Psi^{[0]}(\lambda),
$$

where we have to remember that $\Psi^{[1]}, \Psi^{[2]}, \ldots, \Psi^{[K-1]}$ can (and should) be expressed by $\Psi[0]$ and constants $\kappa_{1}, \ldots, \kappa_{K-1}$. Thus we can use a more compact notation:

$$
\Psi^{[K]}(\lambda)=D_{\left[\Psi[0], \kappa_{1}, \kappa_{2}, \ldots, \kappa_{K-1}\right]}^{[K]}(\lambda),
$$

but the explicit expression for $D^{[K]}$ is extremely complicated. The above notation can be shortened into the following, more compact, form:

$$
\begin{aligned}
& \Psi^{[1]}(\lambda)=D_{[0] 1}(\lambda) \Psi^{[0]}(\lambda), \\
& \Psi^{[2]}(\lambda)=D_{[1] 2}(\lambda) \Psi^{[1]}(\lambda) \\
& \ldots \ldots \ldots \ldots \ldots, \\
& \Psi[K](\lambda)=D_{[K-1] K}(\lambda) \Psi^{[K-1]}(\lambda) .
\end{aligned}
$$

The index [0] may be often omitted. We have, for example:

$$
\begin{aligned}
& \Psi^{[0]}(\lambda) \equiv \Psi(\lambda) \\
& \Psi^{[1]}(\lambda)=D_{[0] 1}(\lambda) \Psi^{[0]}(\lambda)=D(\lambda) \Psi(\lambda)=D^{[1]}(\lambda) \Psi^{[0]}(\lambda), \\
& \Psi^{[2]}(\lambda)=D_{[1] 2}(\lambda) \Psi^{[1]}(\lambda)=D_{[1] 2}(\lambda) D_{[0] 1}(\lambda) \Psi^{[0]}(\lambda)=D^{[2]}(\lambda) \Psi[0](\lambda), \\
& \Psi^{[3]}(\lambda)=D_{[2] 3}(\lambda) D_{[1] 2}(\lambda) D_{[0] 1}(\lambda) \Psi^{[0]}(\lambda)=D^{[3]}(\lambda) \Psi^{[0]}(\lambda)
\end{aligned}
$$

where

$$
D(\lambda)=D_{[0] 1}(\lambda)=\mathbf{e}_{4}\left(\lambda \hat{n}_{1}+\kappa_{1} \hat{p}_{1}\right)
$$


and $\hat{p}_{j}, \hat{n}_{j}$ are defined by

$$
p_{j}+i n_{j}:=\Psi\left(i \kappa_{j}\right)\left(p_{0 j}+i n_{0 j}\right) \Psi^{-1}\left(i \kappa_{j}\right) \quad(j \in \mathbb{N}) .
$$

Theorem 4. Two-fold Darboux transformation of the function $\Psi(\lambda)$ is given by $\Psi^{[2]}(\lambda)=$ $D^{[2]}(\lambda) \Psi(\lambda)$, and $D^{[2]}$ can be expressed by $\kappa_{1}, \kappa_{2}, \hat{p}_{1}, \hat{p}_{2}, \hat{n}_{1}$ and $\hat{n}_{2}$ in the form explicitly symmetric with respect to exchange of indices:

$$
D^{[2]}(\lambda)=\frac{C(\lambda)-\left(\kappa_{1}^{2}-\kappa_{2}^{2}\right) D_{[0] 1}(\lambda) \wedge D_{[0] 2}(\lambda)}{M},
$$

where $D_{[0] j}(\lambda)=\mathbf{e}_{4}\left(\lambda \hat{n}_{j}+\kappa_{j} \hat{p}_{j}\right)(j=1,2)$ and

$$
\begin{aligned}
& M^{2}:=4 \kappa_{1}^{2} \kappa_{2}^{2}\left(\cos ^{2} \varphi+\cos ^{2} \psi\right)-4 \kappa_{1} \kappa_{2}\left(\kappa_{1}^{2}+\kappa_{2}^{2}\right) \cos \varphi \cos \psi+\left(\kappa_{1}^{2}-\kappa_{2}^{2}\right)^{2}, \\
& C(\lambda):=\kappa_{1} \kappa_{2} \cos \varphi\left(2 \lambda^{2}+\kappa_{1}^{2}+\kappa_{2}^{2}\right)-\cos \psi\left(2 \kappa_{1}^{2} \kappa_{2}^{2}+\lambda^{2}\left(\kappa_{1}^{2}+\kappa_{2}^{2}\right)\right) .
\end{aligned}
$$

Proof. We are going to express in a symmetric form $D^{[2]}(\lambda)=D_{[1] 2}(\lambda) D_{[0] 1}(\lambda)$. Note that

$$
\Psi^{[1]}\left(i \kappa_{2}\right)=D_{[0] 1}\left(i \kappa_{2}\right) \Psi\left(i \kappa_{2}\right)=\mathbf{e}_{4}\left(i \kappa_{2} \hat{n}_{1}+\kappa_{1} \hat{p}_{1}\right) \Psi\left(i \kappa_{2}\right),
$$

and

$$
\left(D_{[0] 1}(\lambda)\right)^{-1}=\frac{\lambda \hat{n}_{1}+\kappa_{1} \hat{p}_{1}}{\lambda^{2}+\kappa_{1}^{2}} \mathbf{e}_{4}
$$

Then

$$
D_{[1] 2}(\lambda)=\mathbf{e}_{4}\left(\lambda \hat{n}+\kappa_{2} \hat{p}\right),
$$

where we still use notation (29), but (within this proof) $n$ and $p$ are associated with the matrix $D_{[1] 2}$, i.e.,

$$
p+i n=\Psi^{[1]}\left(i \kappa_{2}\right)\left(p_{02}+i n_{02}\right)\left(\Psi^{[1]}\left(i \kappa_{2}\right)\right)^{-1} .
$$

Therefore, substituting (45),

$$
p+i n=\mathbf{e}_{4}\left(\kappa_{1} \hat{p}_{1}+i \kappa_{2} \hat{n}_{1}\right) \Psi\left(i \kappa_{2}\right)\left(p_{02}+i n_{02}\right) \Psi\left(i \kappa_{2}\right)^{-1}\left(\frac{\kappa_{1} \hat{p}_{1}+i \kappa_{2} \hat{n}_{1}}{\kappa_{1}^{2}-\kappa_{2}^{2}}\right) \mathbf{e}_{4},
$$

which can be rewritten as (compare (29))

$$
p+i n=\frac{\sqrt{p_{2}^{2}}}{\kappa_{1}^{2}-\kappa_{2}^{2}} \mathbf{e}_{4}\left(\kappa_{1} \hat{p}_{1}+i \kappa_{2} \hat{n}_{1}\right)\left(\hat{p}_{2}+i \hat{n}_{2}\right)\left(\kappa_{1} \hat{p}_{1}+i \kappa_{2} \hat{n}_{1}\right) \mathbf{e}_{4},
$$

or

$$
p+i n=\sqrt{p_{2}^{2}} \mathbf{e}_{4}\left(\kappa_{1} \hat{p}_{1}+i \kappa_{2} \hat{n}_{1}\right)\left(\hat{p}_{2}+i \hat{n}_{2}\right)\left(\kappa_{1} \hat{p}_{1}+i \kappa_{2} \hat{n}_{1}\right)^{-1} \mathbf{e}_{4}^{-1},
$$

which is a similarity transformation and can be interpreted as an orthogonal transformation in the (complexified) Clifford algebra. Note that for any Clifford vectors $v, w$ we have

$$
v w v^{-1}=(2\langle v \mid w\rangle-w v) v^{-1}=-w+2 \frac{\langle v \mid w\rangle}{\langle v \mid v\rangle} v .
$$

Therefore

$$
p+i n=\sqrt{p_{2}^{2}} \mathbf{e}_{4}\left(\frac{2\left(\kappa_{1}\left\langle\hat{p}_{1} \mid \hat{p}_{2}\right\rangle-\kappa_{2}\left\langle\hat{n}_{1} \mid \hat{n}_{2}\right\rangle\right)}{\kappa_{1}^{2}-\kappa_{2}^{2}}\left(\kappa_{1} \hat{p}_{1}+i \kappa_{2} \hat{n}_{1}\right)-\left(\hat{p}_{2}+i \hat{n}_{2}\right)\right) \mathbf{e}_{4} .
$$




$$
\begin{gathered}
p=\frac{\sqrt{p_{2}^{2}}}{\kappa_{1}^{2}-\kappa_{2}^{2}}\left(\left(2 \kappa_{1}^{2}\left\langle\hat{p}_{1} \mid \hat{p}_{2}\right\rangle-2 \kappa_{1} \kappa_{2}\left\langle\hat{n}_{1} \mid \hat{n}_{2}\right\rangle\right) \hat{p}_{1}-\left(\kappa_{1}^{2}-\kappa_{2}^{2}\right) \hat{p}_{2}\right), \\
n=\frac{\sqrt{p_{2}^{2}}}{\kappa_{1}^{2}-\kappa_{2}^{2}} \mathbf{e}_{4}\left(\left(2 \kappa_{1} \kappa_{2}\left\langle\hat{p}_{1} \mid \hat{p}_{2}\right\rangle-2 \kappa_{2}^{2}\left\langle\hat{n}_{1} \mid \hat{n}_{2}\right\rangle\right) \hat{n}_{1}-\left(\kappa_{1}^{2}-\kappa_{2}^{2}\right) \hat{n}_{2}\right) .
\end{gathered}
$$

Now we can easily compute $p^{2}, n^{2}$ and then $\hat{p}, \hat{n}$. Let us denote

$$
\cos \varphi:=\left\langle\hat{p}_{1} \mid \hat{p}_{2}\right\rangle, \quad \cos \psi:=\left\langle\hat{n}_{1} \mid \hat{n}_{2}\right\rangle .
$$

Then

$$
p^{2}=n^{2}=\frac{p_{2}^{2} M^{2}}{\left(\kappa_{1}^{2}-\kappa_{2}^{2}\right)^{2}}
$$

where $M^{2}$ is computed in the straightforward way (taking into account $\left\langle\hat{p}_{j} \mid \hat{n}_{k}\right\rangle=0$ ):

$$
M^{2}:=4 \kappa_{1}^{2} \kappa_{2}^{2}\left(\cos ^{2} \varphi+\cos ^{2} \psi\right)-4 \kappa_{1} \kappa_{2}\left(\kappa_{1}^{2}+\kappa_{2}^{2}\right) \cos \varphi \cos \psi+\left(\kappa_{1}^{2}-\kappa_{2}^{2}\right)^{2} .
$$

Therefore

$$
\begin{gathered}
\hat{p}=\frac{\kappa_{1}^{2}-\kappa_{2}^{2}}{M \sqrt{p_{2}^{2}}} p, \quad \hat{n}=\frac{\kappa_{1}^{2}-\kappa_{2}^{2}}{M \sqrt{p_{2}^{2}}} n, \\
M \hat{p}=2\left(\kappa_{1} \cos \varphi-\kappa_{2} \cos \psi\right) \kappa_{1} \hat{p}_{1}-\left(\kappa_{1}^{2}-\kappa_{2}^{2}\right) \hat{p}_{2}, \\
M \hat{n}=2\left(\kappa_{1} \cos \varphi-\kappa_{2} \cos \psi\right) \kappa_{2} \hat{n}_{1}-\left(\kappa_{1}^{2}-\kappa_{2}^{2}\right) \hat{n}_{2} .
\end{gathered}
$$

Thus

$$
D_{[1] 2}(\lambda)=\frac{2 \kappa_{2} \mathbf{e}_{4}\left(\kappa_{1} \cos \varphi-\kappa_{2} \cos \psi\right)\left(\lambda \hat{n}_{1}+\kappa_{1} \hat{p}_{1}\right)}{M}-\frac{\mathbf{e}_{4}\left(\kappa_{1}^{2}-\kappa_{2}^{2}\right)\left(\lambda \hat{n}_{2}+\kappa_{2} \hat{p}_{2}\right)}{M},
$$

i.e.,

$$
D_{[1] 2}(\lambda)=\frac{2 \kappa_{2}\left(\kappa_{1} \cos \varphi-\kappa_{2} \cos \psi\right)}{M} D_{[0] 1}-\frac{\left(\kappa_{1}^{2}-\kappa_{2}^{2}\right)}{M} D_{[0] 2} .
$$

Now, we can compute $D^{[2]}=D_{[1] 2} D_{[0] 1}$ :

$$
D^{[2]}(\lambda)=\left(\frac{2 \kappa_{2}\left(\kappa_{1} \cos \varphi-\kappa_{2} \cos \psi\right)}{M} D_{[0] 1}-\frac{\left(\kappa_{1}^{2}-\kappa_{2}^{2}\right)}{M} D_{[0] 2}\right) D_{[0] 1} .
$$

Using a general property of the Clifford product of vectors

$$
v w=\langle v \mid w\rangle+v \wedge w
$$

(where the wedge denotes the skew product) we get (43).

Corollary 1. The symmetric form of two-fold Darboux transformation can be considered as yet another proof of Bianchi's permutability theorem [5].

\section{Seed Solutions}

In order to produce exact solution by iterating the Darboux transformation we need some starting point: a seed solution. Below we give two simple examples. 


\subsection{The Trivial Background (Plane)}

The data $\vartheta=0, k_{1}=k_{2}=0$ correspond to the trivial background, i.e., to the plane. The linear system (3) assumes the form

$$
\Psi_{, u}=\frac{1}{2} \lambda \mathbf{e}_{1} \mathbf{e}_{5} \Psi, \quad \Psi_{, v}=\frac{1}{2} \lambda \mathbf{e}_{2} \mathbf{e}_{4} .
$$

Hence

$$
\Psi=e^{\frac{1}{2} \lambda u \mathbf{e}_{1} \mathbf{e}_{5}} e^{\frac{1}{2} \lambda v \mathbf{e}_{2} \mathbf{e}_{4}}
$$

Finally

$$
\left.2 \Psi^{-1} \Psi_{, \lambda}\right|_{\lambda=0}=u \mathbf{e}_{1} \mathbf{e}_{5}+v \mathbf{e}_{2} \mathbf{e}_{4},
$$

Performing the projection (13) we get

$$
\mathbf{r}=P\left(\Psi^{-1} \Psi,\left.\lambda\right|_{\lambda=0}\right)=u \mathbf{e}_{1}+v \mathbf{e}_{2},
$$

\subsection{Cylinder}

One can easily see that $\vartheta=0, k_{2}=0, k_{1} \equiv k=$ const satisfy the system (2). The linear system (3) assumes the form

$$
\begin{aligned}
& \Psi_{, u}=\frac{1}{2} \lambda \mathbf{e}_{1} \mathbf{e}_{5} \Psi \\
& \Psi_{, v}=\frac{1}{2} \mathbf{e}_{2}\left(\lambda \mathbf{e}_{4}-k \mathbf{e}_{3}\right) .
\end{aligned}
$$

$\mathbf{e}_{1} \mathbf{e}_{5}$ commutes with $\lambda \mathbf{e}_{2} \mathbf{e}_{4}-k \mathbf{e}_{2} \mathbf{e}_{3}$ and they do not depend on $u, v$. Therefore $\Psi$ can be easily computed

$$
\Psi=e^{\frac{1}{2} \lambda u \mathbf{e}_{1} \mathbf{e}_{5}} e^{\frac{1}{2} v\left(\lambda \mathbf{e}_{2} \mathbf{e}_{4}-k \mathbf{e}_{2} \mathbf{e}_{3}\right)}
$$

Then

$$
\left(\lambda \mathbf{e}_{2} \mathbf{e}_{4}-k \mathbf{e}_{2} \mathbf{e}_{3}\right)^{2}=-\left(\lambda^{2}+k^{2}\right)
$$

Therefore

$$
\begin{gathered}
\Psi=\left(\cosh \frac{\lambda u}{2}+\mathbf{e}_{1} \mathbf{e}_{5} \sinh \frac{\lambda u}{2}\right)\left(\cos \frac{v \sqrt{\lambda^{2}+k^{2}}}{2}+\frac{\mathbf{e}_{2}\left(\mathbf{e}_{4} \lambda-\mathbf{e}_{3} k\right)}{\sqrt{\lambda^{2}+k^{2}}} \sin \frac{v \sqrt{\lambda^{2}+k^{2}}}{2}\right) \\
\Psi^{-1}(0)=\cos \frac{k v}{2}+\mathbf{e}_{2} \mathbf{e}_{3} \sin \frac{k v}{2} \\
\Psi_{, \lambda}(0)=\frac{1}{2} u \mathbf{e}_{1} \mathbf{e}_{5}\left(\cos \frac{k v}{2}-\mathbf{e}_{2} \mathbf{e}_{3} \sin \frac{k v}{2}\right)+\frac{1}{k} \mathbf{e}_{2} \mathbf{e}_{4} \sin \frac{k v}{2}
\end{gathered}
$$

Then

$$
\left.2 \Psi^{-1} \Psi_{\lambda \lambda}\right|_{\lambda=0}=u \mathbf{e}_{1} \mathbf{e}_{5}+\frac{2}{k} \sin \frac{k v}{2} \cos \frac{k v}{2} \mathbf{e}_{2} \mathbf{e}_{4}-\frac{2}{k} \mathbf{e}_{3} \mathbf{e}_{4} \sin ^{2} \frac{k v}{2}
$$

Finally, using the projection (13), we get the cylinder immersed in $\mathbb{R}^{3}$

$$
\mathbf{r}=u \mathbf{e}_{1}-\frac{1}{k} \mathbf{e}_{3}+\frac{1}{k}\left(\mathbf{e}_{3} \cos k v+\mathbf{e}_{2} \sin k v\right)
$$

\section{Conclusions}

We constructed an iterated Darboux transformation for isothermic surfaces using the Clifford algebra approach. Our main result is a symmetric representation of two-fold Darboux transformation (Theorem 4). Thus we made some progress in the direction of constructing symmetric compact formulas for "multi-soliton" isothermic surfaces, what reduces to transforming $\Psi^{[K]}$ (given by (38)) into a form that is explicitly invariant with respect to permutations of real eigenvalues $\kappa_{1}, \ldots, \kappa_{K}$. Another open problem, more chal- 
lenging, is to find analogous formulas in a direct way and with more general set of complex eigenvalues. We also expect to extend our approach on related multidimensional problems (see, e.g., [32]).

Author Contributions: conceptualization, J.L.C.; methodology, J.L.C.; formal analysis, J.L.C. and Z.H.; investigation, J.L.C. and Z.H.; writing—original draft preparation, J.L.C.; writing—review and editing, J.L.C. All authors have read and agreed to the published version of the manuscript.

Funding: This research received no external funding.

Conflicts of Interest: The authors declare no conflict of interest.

\section{References}

1. Lamé, G. Mémoire sur les surfaces isothermes dans les corps solides homogènes en équilibre de température. J. Math. Pures Appl. 1837, 2, 147-183.

2. Bertrand, J. Mémoire sur les surfaces isothermes orthogonales. J. Math. Pures Appl. 1844, 9, 117-130.

3. Klimczewski, P.; Nieszporski, M.; Sym, A. Luigi Bianchi, Pasquale Calapso and solitons. Rend. Sem. Mat. Messina (Atti del Congresso Internazionale in onore di Pasquale Calapso) 2000, 223-240.

4. Darboux, G. Sur les surfaces isothermiques. C. R. Acad. Sci. Paris 1899, 128, 1299-1305. [CrossRef]

5. Bianchi, L. Ricerche sulle superficie isoterme e sulle deformazione delle quadriche. Ann. Matem. 1905, 11, 93-157. [CrossRef]

6. Novikov, S.; Manakov, S.V.; Pitaevskii, L.P.; Zakharov, V.E. Theory of Solitons. The Inverse Sattering Method; Springer: New York, NY, USA, 1984.

7. Cieśliński, J.; Goldstein, P.; Sym, A. Isothermic surfaces in $E^{3}$ as soliton surfaces. Phys. Lett. A 1995, 205, 37-43. [CrossRef]

8. Bobenko, A.; Pinkall, U. Discrete isothermic surfaces. J. Reine Angew. Math. 1996, 475, 187-208.

9. Burstall, F.; Hertrich-Jeromin, U.; Pedit, F.; Pinkall, U. Curved flats and isothermic surfaces. Math. Z. 1997, 225, 199-209. [CrossRef]

10. Hertrich-Jeromin, U.; Pedit, F. Remarks on the Darboux transform of isothermic surfaces. Doc. Math. 1997, 2, 313-333.

11. Musso, E.; Nicolodi, L. Special isothermic surfaces and solitons. Contemp. Math. 2001, 288, 129-148.

12. Burstall, F.E. Isothermic surfaces: Conformal geometry, Clifford algebras and integrable systems. AMS/IP Stud. Adv. Math. 2006, $36,1-82$.

13. Burstall, F.E.; Hertrich-Jeromin, U. The Ribaucour transformation in Lie sphere geometry. Differ. Geom. Appl. 2006, 24, 503-520. [CrossRef]

14. Burstall, F.; Hertrich-Jeromin, U.; Müller, C.; Rossman, W. Semi-discrete isothermic surfaces. Geom. Dedicata 2016, 183, 43-58. [CrossRef]

15. Tafel, J. Covariant Description of Isothermic Surfaces. Rep. Math. Phys. 2016, 78, 295-303. [CrossRef]

16. Hertrich-Jeromin, U.; Honda, A. Minimal Darboux transformations. Beitr. Algebra Geom. 2017, 58, 81-91. [CrossRef]

17. Cieśliński, J.L.; Kobus, A. Group interpretation of the spectral parameter. The case of isothermic surfaces. J. Geom. Phys. 2017, 113, 28-37. [CrossRef]

18. Fuchs, A. Transformations and singularities of polarized curves. Ann. Glob. Anal. Geom. 2019, 55, 529-553. [CrossRef]

19. Corro, A.M.V.; Ferro, M.L. New Isothermic surfaces. arXiv 2020, arXiv:2011.07941v1.

20. Cieśliński, J.L. A class of linear spectral problems in Clifford algebras. Phys. Lett. A 2000, 267, 251-255. [CrossRef]

21. Cieśliński, J.L. The Darboux-Bäcklund transformation without using a matrix representation. J. Phys. A Math. Gen. 2000, 33, L363-L368. [CrossRef]

22. Bobenko, A.I.; Hertrich-Jeromin, U.J. Orthogonal nets and Clifford algebras. arXiv 1998, arXiv:math/9802126.

23. Cieśliński, J. The Darboux-Bianchi transformation for isothermic surfaces. Classical results versus the soliton approach. Diff. Geom. Appl. 1997, 7, 1-28. [CrossRef]

24. Sym, A. Soliton surfaces and their applications. In Geometric Aspects of the Einstein Equations and Integrable Systems; Lecture Notes in Physics; Martini, R., Ed.; Springer: Berlin, Germany, 1985; Volume 239, pp. 154-231.

25. Lounesto, P. Clifford Algebras and Spinors; Cambridge University Press: Cambridge, UK, 1997.

26. Vaz, J.; da Rocha, R. An Introduction to Clifford Algebras and Spinors; Oxford University Press: Oxford, UK, 2016.

27. Cieśliński, J. An algebraic method to construct the Darboux matrix. J. Math. Phys. 1995, 36, 5670-5706. [CrossRef]

28. Gu, C.H. Bäcklund Transformations and Darboux Transformations. In Soliton Theory and Its Applications; Springer: Berlin, Germany, 1995; pp. 122-151..

29. Mikhailov, A.V. The reduction problem and the inverse scattering method. Phys. D Nonlinear Phenom. 1981, 3, 73-117. [CrossRef]

30. Cieśliński, J.L. Algebraic construction of the Darboux matrix revisited. J. Phys. A Math. Theor. 2009, 42, 404003. [CrossRef]

31. Biernacki, W.; Cieśliński, J.L. A compact form of the Darboux-Bäcklund transformation for some spectral problems in Clifford algebras. Phys. Lett. A 2001, 288, 167-172. [CrossRef]

32. Cieśliński, J.L. Geometry of submanifolds derived from Spin-valued spectral problems. Theor. Math. Phys. 2003, 137, 1394-1403. [CrossRef]

33. Matveev, V.B.; Salle, M.A. Darboux Transformations and Solitons; Springer: Berlin/Heidelberg, Germany, 1991. 
34. Rogers, C.; Schief, W.K. Bäcklund and Darboux Transformations. Geometry and Modern Applications in Soliton Theory; Cambridge University Press: Cambridge, UK, 2002.

35. Sakhnovich, A.L. Generalized Bäcklund-Darboux Transformation: Spectral Properties and Nonlinear Equations. J. Math. Anal. Appl. 2001, 262, 274-306. [CrossRef]

36. Sakhnovich, A.L.; Sakhnovich, L.A.; Roitberg, I.Y. Inverse Problems and Nonlinear Evolution Equations; De Gruyter: Berlin, Germany, 2013.

37. Cieśliński, J.L.; Biernacki, W. A new approach to the Darboux-Bäcklund transformation versus the standard dressing method. J. Phys. A Math. Gen. 2005, 38, 9491-9501. [CrossRef] 